\title{
Quadrant Asymmetric Design Contact Lens for Visual Rehabilitation after Eye Trauma
}

\author{
Nir Erdinest ${ }^{a, b} \quad$ Ortal Palatchi Sabag ${ }^{c} \quad$ Naomi London ${ }^{d}$ \\ Abraham Solomon ${ }^{a}$ \\ aDepartments of Ophthalmology, Hadassah-Hebrew University Medical Center, Jerusalem, \\ Israel; 'bThe Keratoconus Center, Jerusalem, Israel; 'Soflex CooperVision Specialty Eyecare \\ Division, CooperVision Israel Ltd., D.N. Misgav, Israel; dPrivate Clinic, Jerusalem, Israel
}

\section{Keywords}

Quadrant asymmetric · Contact lens · Eye trauma · Peripheral design · Visual rehabilitation after

\begin{abstract}
The purpose of this case report is to demonstrate the efficacy of an asymmetric peripheral design scleral contact lens in a case of highly irregular corneal-scleral pattern due to trauma. A 63-year-old patient was involved in a jeep accident which caused a partial-thickness penetrating injury to the peripheral cornea of his left eye. The subsequent corneal irregularity extended beyond the limbus into the sclera which made it difficult to stabilize a contact lens. A quadrant specific peripheral curve (quadrant asymmetric periphery) scleral contact lens successfully resulted in improved comfort and visual acuity. This is the first known published case to use this lens design to correct a post-trauma irregular cornea-scleral relationship. Quadrant asymmetric periphery scleral contact lenses can be effective in cases of severe irregular corneal-scleral patterns.
\end{abstract}

(C) 2021 The Author(s).

Published by S. Karger AG, Basel

\section{Introduction}

Corneal lacerations may induce severe corneal deformities, scars, and damage to surrounding tissues such as the limbus, sclera, and iris [1,2]. Moreover, full-thickness wounds, corneal trauma, and corneal perforation can lead to severe long-term complications [1, 2]. Patients who develop corneal scars in the visual axis will generally be treated with a corneal transplant [1]. Irregular astigmatism resulting from corneal deformity may eventually require corneal rigid gas permeable (RGP) contact lenses or, in more extreme cases, scleral contact 
lenses to improve quality of vision [1]. In cases of trauma that involve the peripheral cornea and iris dislocation, fitting a lens can be very challenging as the asymmetry can extend well into the sclera, making a lens stabilization difficult. In this case report, designing a scleral lens with a quadrant asymmetric periphery solved this type of irregularity.

\section{Case Report}

A 63-year-old male was injured in a motor vehicle accident approximately 30 years ago. The accident occurred during a military exercise in a jeep. The nature of the exercise necessitated the patient did not wear a helmet or seatbelt, and he was thrown through the window, injured by the broken glass. A partial-thickness corneal laceration in his left eye was incurred which led to corneal, limbal, and scleral scars in the superior and inferior tissue as well as pupil dislocation. A year prior to the scleral fitting examination, the patient contracted epidemic keratoconjunctivitis that left residual infiltrative scarring which further adversely affected the vision of his left eye.

Best spectacle-corrected visual acuity at the initial visit was $6 / 10+$ with $+1.00 \mathrm{D}$ in the right eye and hand motion at $4 \mathrm{~m}$ with $+4.00 /-7.00 \times 60$ in his left eye which improved with pinhole to $6 / 10$ and $6 / 12$, respectively. Corneal topography of the left eye demonstrated significant irregularity of the cornea with significant inferior steepening and superior flattening (shown in Fig. 1). Patients' left eye the inferior-superior (I-S) value was $1.7 \mathrm{D}$, the surface asymmetry index value was 2.31 , and the surface regularity index value was 1.44 . Biomicroscopy showed a superior corneal scar (around $120-130^{\circ}$ ) involving the sclera as

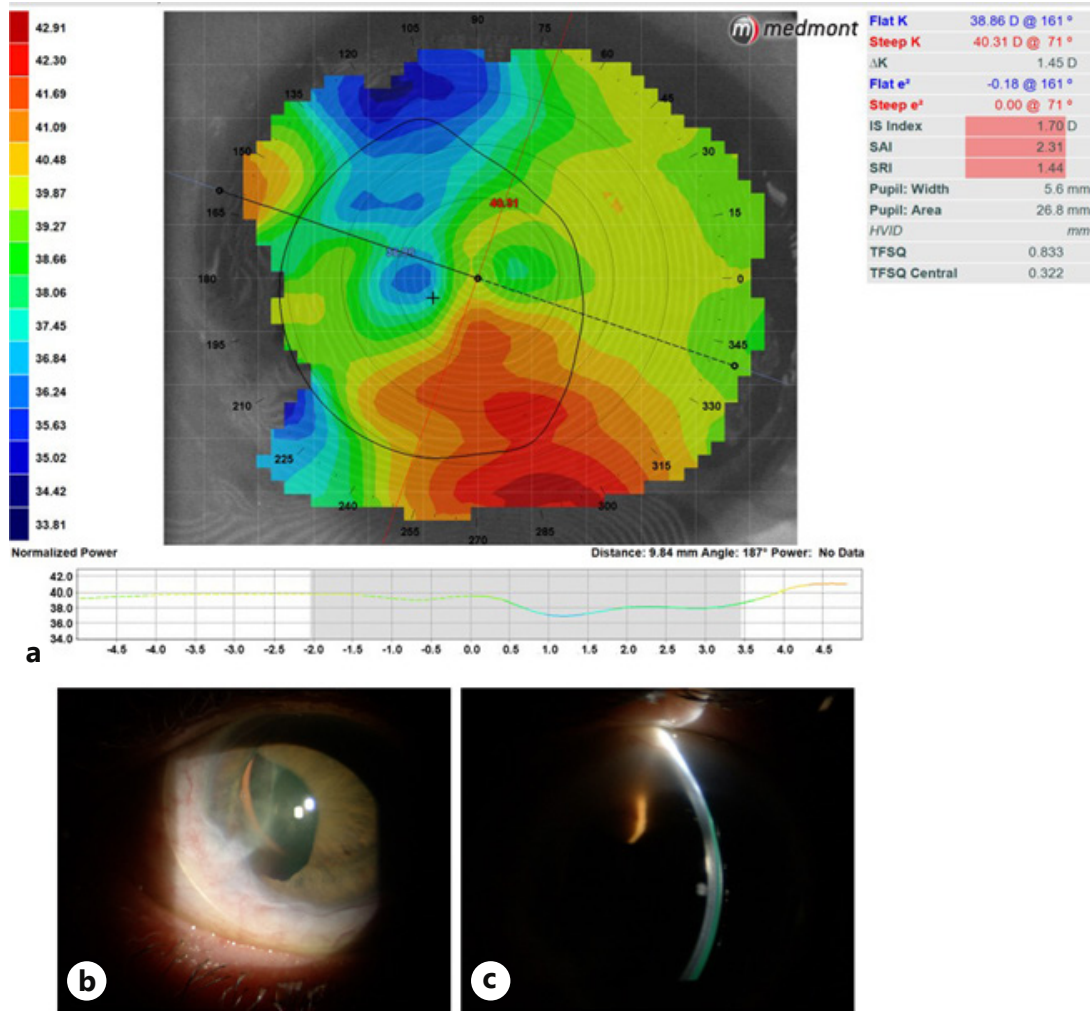

Fig. 1. Corneal topography and slit-lamp examination images. OS corneal topography (a). Wide beam reveals anterior synechia and scars, iridodialysis, an irregular and fixed pupil (b). Vertical optic section of the cornea, central clearance was 200 microns, vault after $8 \mathrm{~h}$ (c). 
well. In addition, there was a scar in the inferior periphery of the cornea extending to the limbus at $210^{\circ}$, subepithelial infiltrates as a result of epidemic keratoconjunctivitis. There was a distorted and eccentric pupil, drawn toward the infero-nasal periphery. The patient is phakic and does not suffer from photophobia.

The patient had attempted numerous corneal RGP fitting designs at other practitioners including Rose K2, but he complained of discomfort and insufficient improvement in visual acuity. In addition, most of the lenses ejected from the patient's eyes. From the practitioners' perspective, none of these lenses achieved acceptable stability or centration. Hybrid lenses as well as a softback toric lens helped with patient comfort but still did not achieve adequate visual acuity. Prior to scleral lens fitting an endothelial cell count was performed and measured above 1,000 cells $/ \mathrm{mm}$ which is acceptable for scleral lens wear [3]. There are several scleral profilometers that help identify the scleral structures and design better asymmetric periphery lenses. The topographer in this clinic, the MedmontE300 (Medmont, Nunawading, Australia), was unable to capture a full map of the sclera due to the advanced warpage (shown in Fig. 1a).

The patient presented with a superior corneal scar involving the sclera as well as an inferior periphery corneal scar (shown in Fig. 1b). The irregular structure of the limbus and sclera area became apparent upon lens insertion in the form of a gap between the landing zone of the lens and the sclera. A few attempts to fit spherical as well as toric periphery designed lenses were made, but they all failed as air bubbles became trapped under the lens. An asymmetric peripheral design allowed the lens to vault the distorted structures, land acceptably on the sclera, and provided comfort and rotational stability. The lens chosen was the Irregular Corneal Design FlexFit (Katt Design group Ltd, Canada) 16.3 diameter scleral lens made from Paragon HDS $^{\circledR}$ (paflufocon B) material. This lens gives the practitioner the option to adjust the height of each peripheral quadrant individually, providing an excellent solution in cases of scleral asymmetry (shown in Fig. 2). This is designed by utilizing the information available from the topography maps combined with clinical observation and requesting the desired sagittal height for each quadrant of the lens individually, in microns units. A scleral lens was designed with a decrease in the peripheral zone in quadrant Q2 $\left(130^{\circ}\right)$ of 150 microns from the standard vault in order to reduce the gap between the lens and cornea (shown in Fig. 1c). This still allowed for contact lens clearance over the scar area. The clearance was reduced in Q1, Q3, and Q4 by 75, 25, and 25 microns, respectively.

The additional final lens parameters included a diameter of $16.3 \mathrm{~mm}, 4,100$ microns of sagittal height, an elevated peripheral central clearance zone from the standard by 75 microns over $360^{\circ}$.

Contact lens fitting was evaluated via slit lamp and optical coherence tomography (OCT). The OCT demonstrated acceptable clearance of 172 microns at the corneal apex after $8 \mathrm{~h}$ of wear (shown in Fig. 3).

The scleral contact lens exhibited excellent centration. Following the quadratic scleral lens fitting, the patient's best-corrected visual acuity in his left eye dramatically improved from hand motion to $6 / 12$, and his near acuity improved to Jaeger 1 with $+3.00 \mathrm{D}$ reading

Fig. 2. Scleral quadrant asymmetric peripheral design. Scleral quadrant (Q) asymmetric peripheral design (Focal Point software, Advance Medical, Milano, Italy v 7.0). Q1 and Q2 represent quadrants 1 and 2, respectively.

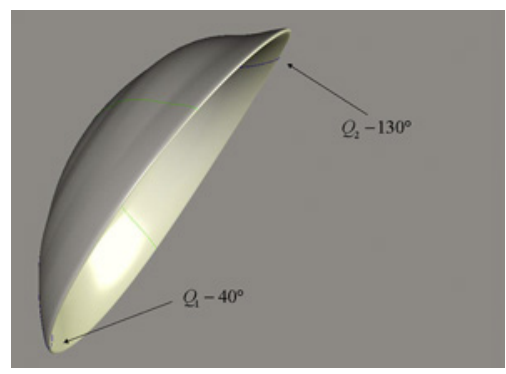




\section{Corneal Six-Radial Line}

Scan Width: $6 \mathrm{~mm}$
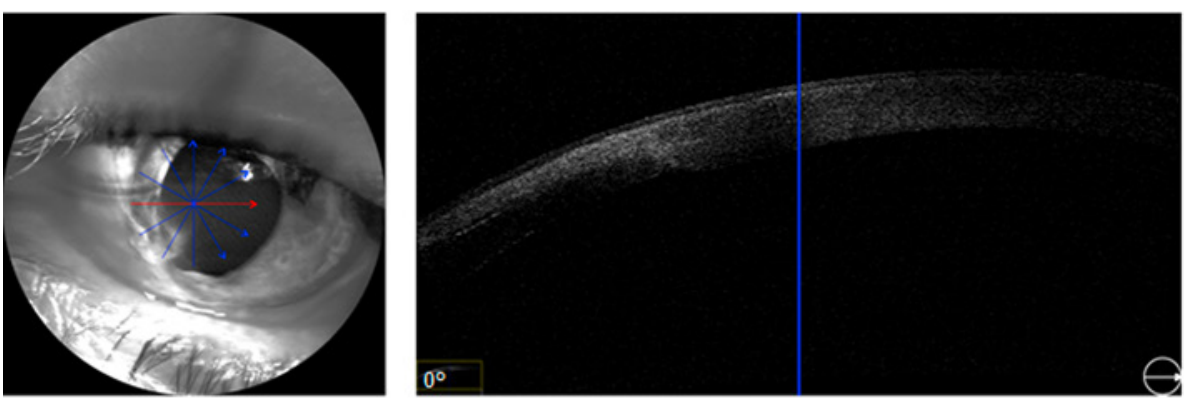

Iris Image OCT Image
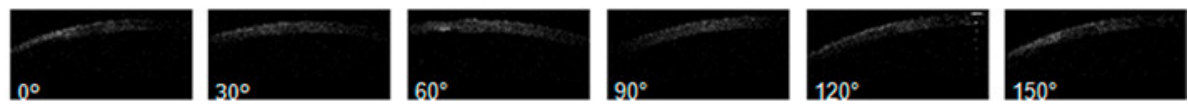

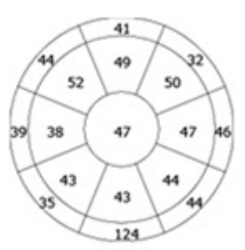

Epithelium Thickness $(\mu \mathrm{m})$

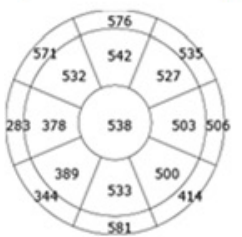

Corneal Thickness $(\mu \mathrm{m})$

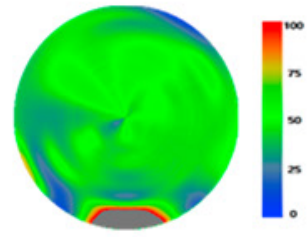

Epithelium Thickness $(\mu \mathrm{m})$

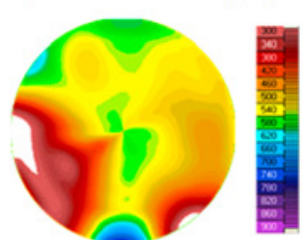

Corneal Thickness $(\mu \mathrm{m})$

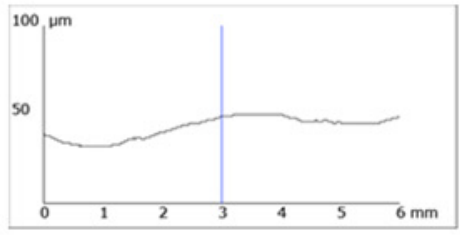

Epithelium Sample:3.00mm Thickness:49um

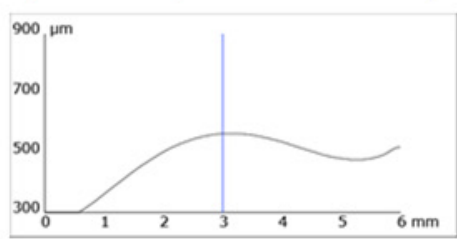

Corneal Sample:3.00mm Thickness:566 $\mu \mathrm{m}$

a

Anterior HD Line

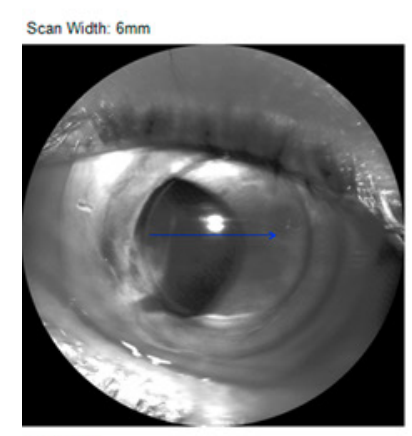

Inis Image

oct Image

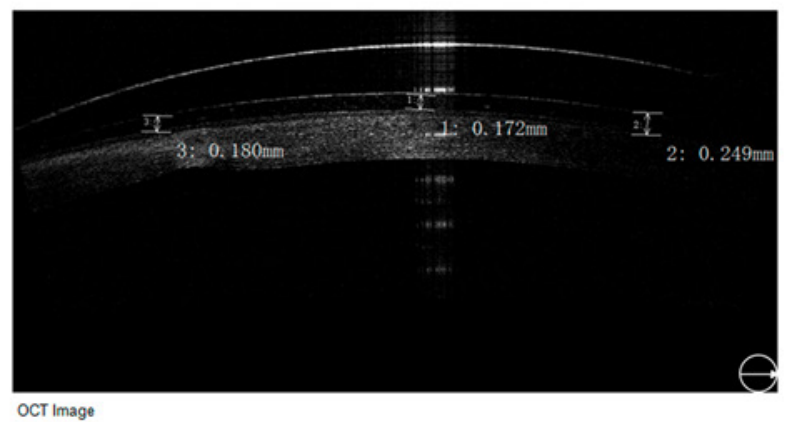

Doctor's Signature:

b

Fig. 3. OCT examination. OCT corneal six-radial line characteristics and corneal pachymetry (a). OCT measurements of scleral contact lens show 172 microns apical clearance after $10 \mathrm{~h}$ of contact lens wear (b).

spectacles worn over the contact lens. The patient achieved improved visual acuity and comfort. The scleral lens with quadrant asymmetric peripheral design was well tolerated for 12-16 h of daily wear (refreshing with nonpreserved saline every $4 \mathrm{~h}$ ) without complaints or 
complications. He disinfected the lens using a one-step hydrogen peroxide solution. The patient returned for follow-up visits a week, a month, at 3 months, at 6 months, and 1 year after dispensing. Visual acuity was stable, assessment of fit continued to show corneal vaulting with no pressure on the scar or excessive pressure on the other ocular surface tissue. Fluorescein and lissamine green staining were performed to ensure ocular health was intact. There was no limbal or bulbar hyperemia and no noted tarsal giant papillary conjunctivitis. There were no discharges or debris on the lens.

\section{Discussion}

One of the possible injuries resulting from a motor vehicle accident when the driver does not wear a seatbelt is corneal trauma and lacerations, in this case from a window fragment. A corneal laceration is one of the more extreme of eye wounds and can leave a permanent partial or full-thickness corneal lysis $[1,2,4,5]$. These can result in severe deformities, scars, and damage to surrounding tissues such as the limbus, sclera, and pupil. The cornea and the pupil are integral components of the visual axis and any damage to these structures decreases the quality of vision and may lead to permanent visual loss [1,2]. Moreover, corneal trauma, corneal perforation, or any ocular full-thickness wound can induce severe long-term complications such as intraocular infections and ocular hypotension $[1,2]$.

Full-thickness corneal lacerations are treated as open globe wound while partialthickness corneal lacerations are treated with the same protocol as foreign bodies, namely broad-spectrum antibiotics $[1,2]$. Partial thickness, also known as non-penetrating lacerations, also requires a thorough washout of the wound $[1,6]$. Wounds $<2 \mathrm{~mm}$ in size can be sealed with fibrin glue, a preferred method to sutures to avoid further injury and points of possible infection $[1,4,6,7]$.

Patients who develop severe corneal scars in the visual axis may need a corneal transplant [1]. Irregular astigmatism resulting from corneal deformity may eventually require RGP contact lenses or in severe cases, scleral contact lenses to improve vision [1]. In this case report, the ocular trauma was not a full-penetrating laceration but did deform the cornea and sclera with a scar decreasing the vision so severely as to require a scleral lens with quadrant asymmetric periphery. Scleral contact lens wear is an effective tool for providing visual rehabilitation in severe ocular surface diseases including eye trauma, as was demonstrated in this case study [8].

The unique challenge of this particular case was the incongruent scar tissue deformities in the cornea, limbus, and sclera necessitating distinct adjustments to the corneal sagittal height, the limbal vault as well as each quadrant in the periphery. Each of these alterations further affects the other parameters. This uncommon topography caused by trauma, necessitating numerous individually tailored adjustments to provide a successful solution, to the best of our knowledge has not yet been published in the literature.

Asymmetric peripheral scleral lenses enable various sagittal depths around the limbus as well as throughout the landing zone on the sclera $[9,10]$. Designing the lens is accomplished by combined assessment of the corneal topography map, contact lens in vivo fluorescein pattern, and an OCT map. Further adjustments can be made if required after observing the fluorescein pattern and OCT while the lens is on the eye at follow-up visits. This final lens provides rotational stability, improves comfort, and provides a solution for irregular corneas. Customized shaping improves the quality of vision as well by decreasing astigmatic and higher order aberrations [8].

In conclusion, scleral lenses with quadrant asymmetric peripheral design can play an important role in providing successful visual rehabilitation and a therapeutic solution for 
singular ocular conditions including severe corneal abnormalities, but this unique design would be attempted only after conventional contact lenses, spherical, or toric periphery scleral lenses and medical treatment have failed and surgery is undesirable or contraindicated.

\section{Acknowledgements}

Dr. Nir Erdinest is grateful to the Azrieli Foundation for the award of postdoc Azrieli Fellowship and Hadassah-Hebrew University Medical Center for the award of postdoctoral fellows.

\section{Statement of Ethics}

The authors state that this case report was conducted according to good clinical practice and have no ethical conflicts to disclose. The patient consented to publication of the case report and any accompanying images in writing. This report does not contain any personal information that could lead to the identification of the patient.

\section{Conflict of Interest Statement}

Financial disclosures: N.E. counselor for CooperVision Israel Ltd. O.P.S. works at CooperVision Israel Ltd. The following authors have no financial disclosures: N.L. and A.S. Authorship: all the authors attest that they meet the current ICMJE criteria for authorship.

\section{Funding Sources}

Financial disclosures for this study were CooperVision Israel Ltd., Soflex CooperVision Specialty Eyecare Division, Bar-Lev Industrial Park, and D.N. Misgav, Israel.

\section{Author Contributions}

N.E.: patient examination, contact lens fitting and follow-up, interpretation of the data, and manuscript preparation. O.P.S.: patient contact lens fitting, data collection, and patient follow-up. N.L.: patient follow-up, data collection, and manuscript preparation. A.S.: involved in the design, revision, and conduct of the study. All the authors approve the final version of the manuscript for publication.

\section{References}

1 Kuhn F, Pieramici DJ. Ocular trauma: principles and practice. New York, NY: Thieme; 2002.

2 Yan H. Mechanical ocular trauma: current consensus and controversy. Springer; 2016.

3 Van der Worp E. A guide to scleral lens fitting, version 2.0 [monograph online]. Forest Grove, OR: Pacific University; 2015.

4 Armstrong GW, Stefater JA, Yonekawa Y. Case 2: linear corneal laceration from broken glass. Management of open globe injuries. Springer; 2018. p. 73-80.

\section{Karger'}


5 Shoji MK, Tran AQ, Nikpoor N, Lee WW. Corneal laceration associated with upper eyelid blepharoplasty. Ophthalmic Plast Reconstr Surg. 2020;36(1):e21-3.

6 Wolkow N, Shah AS, Grob S. Case 1: linear corneal laceration from scissors. Management of open globe injuries. Springer; 2018. p. 61-72.

7 Rao R, Miller JB, Grob S. Case 5: limbus to limbus corneal laceration from nail gun injury. Management of open globe injuries. Springer; 2018. p. 103-11.

8 van der Worp E, Bornman D, Ferreira DL, Faria-Ribeiro M, Garcia-Porta N, González-Meijome JM. Modern scleral contact lenses: a review. Cont Lens Anterior Eye. 2014;37(4):240-50.

9 Bergmanson JP, Ezekiel DF, van der Worp E. Scleral contact lenses and hypoxia: theory versus practice. Cont Lens Anterior Eye. 2015;38(3):145-7.

10 Ritzmann M, Caroline PJ, Börret R, Korszen E. An analysis of anterior scleral shape and its role in the design and fitting of scleral contact lenses. Cont Lens Anterior Eye. 2018;41(2):205-13. 